\title{
An unusual case presentation of spontaneous coronary artery dissection
}

\author{
Sudeep Thumma ${ }^{1}$, Jaya Bathina ${ }^{2 *}$, Satish Madiraju ${ }^{2}$, David H. Spodick², Prasad Maddukuri ${ }^{2}$ \\ ${ }^{1}$ Department of Medicine, Saint Francis Hospital, Hartford, USA \\ ${ }^{2}$ Department of Cardiology and Medicine, Saint Vincent Hospital, Worcester, USA \\ Email: ${ }^{*}$ jaya.bathina@stvincenthospital.com
}

Received 13 May 2012; revised 11 July 2012; accepted 21 July 2012

\begin{abstract}
Spontaneous coronary artery dissection is a rare cause of acute myocardial infarction in the young which can be life threatening. The clinical presentation is wide, ranging from simple chest pain to sudden death. Asymptomatic spontaneous coronary dissection is extremely rare. We present a case of asymptomatic spontaneous coronary artery dissection which was managed conservatively; we also review the literature and various treatment options.
\end{abstract}

Keywords: Spontaneous Coronary Artery Dissection; Ischemic Cardiomyopathy

\section{INTRODUCTION}

Spontaneous coronary artery dissection (SCAD) is a rare cause of acute myocardial infarction in the young which can be life threatening. It commonly involves a single coronary vessel. However, multi-vessel dissection involvement [1] has been identified which generally requires intervention. Asymtomatic cases are rare and are diagnosed incidentally [2,3]. We present a case of asymptomatic SCAD which was managed conservatively; we also review the literature and various treatment options.

\section{CASE}

A 53-year old woman with a history of dyslipidemia and hyperhomocystenemia presented to the hospital with a few minute history of slurred speech consistent with transient ischemic attack. Vitals on admission were stable and physical examination was unremarkable. Carotid duplex was unrevealing for obstruction and an electrocardiogram demonstrated sinus rhythm and no evidence of prior infarction. An echocardiogram revealed a left ventricular ejection fraction (LVEF) of $15 \%$ and severe pulmonary hypertension. Left heart catheterization showed dissection in the left anterior descending coronary artery

${ }^{*}$ Corresponding author.
(LAD) with a $90 \%$ ostial stenosis and dissecttion of the left circumflex artery (LCX) (Figure 1). Right and left heart catheterization revealed elevated bilateral ventricle pressures with evidence of severe pulmonary hypertension. A dobutamine stress echocardiogram showed augmentation consistent with hibernating myocardium, except the inferior wall. She was not considered for coronary artery bypass (CABG) as she did not have technically favorable targets due to a small circumflex artery and unknown extent of dissection of the LAD. She was started on optimal medical management for ischemic cardiomyopathy as well as anticoagulation to prevent future embolic events. A computerized tomographic angiogram done 15 days later to access the extent of dissection showed complete resolution of dissection (Figures 2 and 3) with LVEF of 19\%. Echocardiogram performed 3 months later showed no improvement in the LVEF however was symptomatic with shortness of breath. Currently, she is being considered for (automated intracardiac device) AICD placement with thoughts of future for heart transplantation should her systolic dysfunction remain severely depressed.

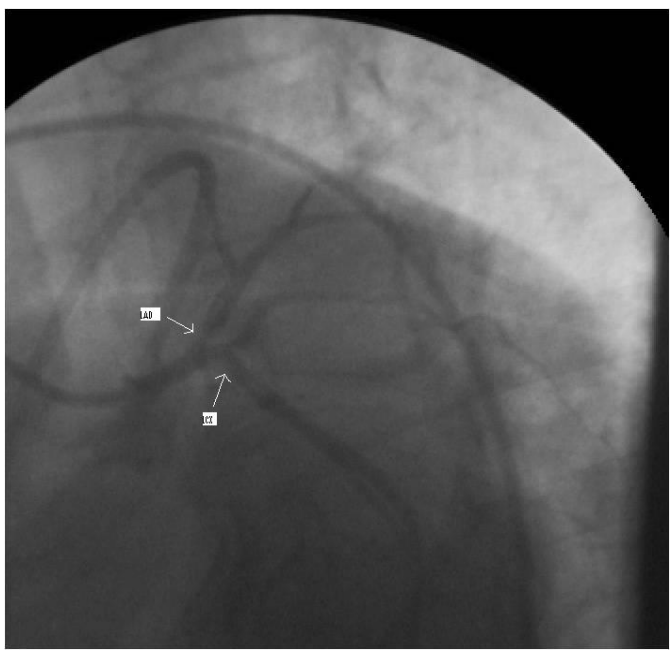

Figure 1. Coronary angiogram showing dissections in proximal LAD and LCX. 


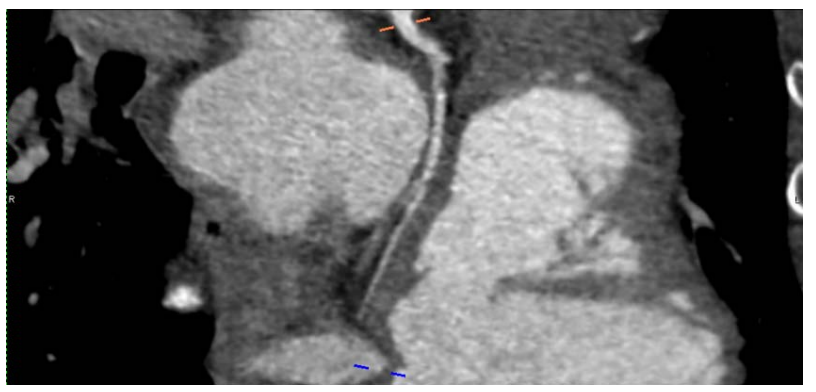

Figure 2. LCX showing resolution of SCAD.

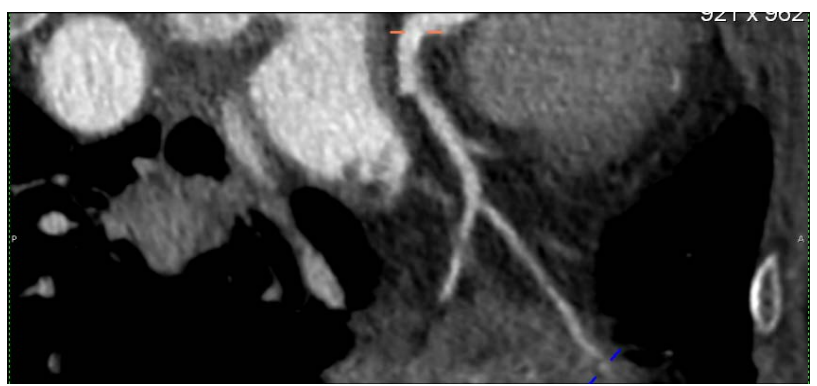

Figure 3. LAD showing resolution of SCAD.

\section{DISCUSSION}

SCAD is an uncommon clinical entity observed in $0.1 \%$ of patients undergoing cardiac catheterization. It was first described in 1931 by Pretty. Since then there are less than 200 cases reported [4]. The true incidence of SCAD is usually underestimated as nearly $70 \%$ of the cases are diagnosed at autopsy [5]. Seventy-five percent of the patients with SCAD are women with a mean age at presentation of 40 [5]. Twenty-five percent of SCAD in women are described during pregnancy and the postpartum period. It has also been described in older patients with or without associated coronary artery disease (CAD). The clinical presentation is wide, ranging from acute coronary syndrome to sudden death. Some of the symptoms include chest pain, dyspnea, syncope, cyanosis and/or vomiting. Pattern and severity of symptoms vary with extent, location, rate of development of the dissection and presence of CAD, which may include ST elevation. Asymptomatic SCAD is extremely rare. An extensive review of PUBMED search revealed only 2 asymptomatic cases published to date (Table 1).

The dissection plane is noted in the outer third of the media or between the media and adventitia as opposed to aortic dissection where the plane is between the intimal and medial layers [5]. The standard test to diagnose SCAD is coronary angiography. The diagnosis is made when contrast media is seen in two lumina separated by a radiolucent intimal flap. Intravascular ultrasound is useful to differentiate atherosclerotic CAD from dissection when in doubt in the coronary angiogram [6]. There are no batteries of tests indicated in guidelines in these patients. SCAD usually involves a single coronary artery; only a few cases of multivessel coronary dissection have been reported. The LAD is commonly involved (80\% of cases) [7]. The LCX is involved in less than $2 \%$ of cases. Left coronary territory is more commonly involved in women; in contrast to right coronary artery which is involved in men [5] the cause for this sex difference is unclear.

Apart from the pregnancy, SCAD has also been described in association with Ehler-Danlos syndrome [8], Marfan's syndrome, Kawasaki's disease, systemic lupus erythematosis [9], hypereosinophilic syndrome, fibro-muscular dysplasia, hypersensitivity angiitis, sarcoidosis, as an extension of aortic dissection, in the setting of atherosclerotic disease, blunt trauma [10], mitral stenosis, and cocaine abuse [11]. There are some case reports associated with exercise, oral contraceptives [12] and auto-immune thyroiditis. Of note it has also been found in healthy individuals without identifiable risk factors as in our patient. A reasonable explaination for SCAD is eosinophilic infiltration, where there is release of lytic agents which cause dissection [7]. Cystic medial necrosis is seen in $37 \%$ of the cases [7]. However pathophysiology varies with the actual cause, such as catecholamine surge and coronary vasospasm in coccaine abuse, shear stress during deccelaration in blunt chest trauma, endocrine changes leading to arterial wall remodeling in pregnancy and women taking oral contraceptives.

There were good results with medical therapy alone (aspirin, nitrates, B blockers, ACE inhibitors) or in combination with coronary angioplasty with stent placement [5]. There is a survival benefit with CABG in SCAD patients like any other acute coronary syndrome patients. CABG has best the results when there is left main, multivessel involvement or in cases where there is recurrent ischemia [5]. The role of thrombolysis is controversial as there are reports where it was successful [13]; at the

Table 1. Asymptomatic spontaneous coronary artery dissection published from 2004-2012.

\begin{tabular}{|c|c|c|c|c|c|c|c|c|}
\hline S. No & Article & Year & $\mathrm{A} / \mathrm{S}$ & V inv. & EF (\%) & Diagnosis & Treatment & Prognosis \\
\hline 1. & Rovner et al. [2] & 2004 & $27 / \mathrm{F}$ & LM, LAD, CX & $45-50$ & Catheterization & CABG & Good \\
\hline 2. & Shankarappa et al. [3] & 2009 & $25 / M$ & LAD & 54 & Catheterization & Conservative & Unknown \\
\hline 3. & Thumma et al. (current case) & 2012 & $53 / \mathrm{F}$ & $\mathrm{LAD}, \mathrm{CX}$ & 15 & Catheterization & Spontaneously resolved & - \\
\hline
\end{tabular}

A—age; S—sex; MV—multi-vessel; SV—single vessel; V—vessel; M—male; F—female; LM—left main; LAD—left anterior descending artery; CX—circumflex; CABG—coronary artery bypass graft. 
same time there are reports which caused extension of dissection due to thrombolysis [14]. There is no data on role of anticoagulation similar to carotid artery dissection. Successful use of glycoprotein IIb/IIIa inhibitors in SCAD has been reported in sporadic cases [15]. Artificial heart implantation and heart transplantation were used in some patients with refractory heart failure. There are cases where there was spontaneous healing of a coronary dissection as in our case. The natural history of dissecttion is unknown; more trails have to be done with follow up for long term to access the course.

Prognosis of these patients is generally poor, with a mean survival of $30 \%$. Some data suggest that there is better survival in men as compared to women. Prognosis depends on the number and types of vessels involved and the extent of dissection. A good long-term prognosis is seen in patients with limited dissection, incomplete obstruction, infarction without complications and residual ischemia at high workload on stress testing.

Asymptomatic presentation of SCAD, as in our case is rare and most of the time goes unnoticed. ACC guidelines suggest angiograms for newly diagnosed cardiomyopathy patients in the setting of chest pain, is it reasonable to recommend in all newly diagnosed patients in order not to miss the diagnosis in patients like this is unclear. Moreover, there is no literature on time for dissecttions to heal and probable role of homocysteine gene mutation in SCAD which have to be studied in future.

\section{REFERENCES}

[1] Cohen, D.E. and Strimike, C.L. (2000) A case of multiple spontaneous coronary artery dissections. Catheterization and Cardiovascular Interventions, 49, 18-20. doi:10.1002/(SICI)1522-726X(200003)49:3<318::AID-C CD19>3.0.CO;2-C

[2] Rover, A., Srihari, T., Rogers, J., Moazami, N. and Lasala, J. (2004) Spontaneous multivessel coronary artery dissection in a young asymptomatic patient. Journal of Interventional Cardiology, 17, 123-127.

[3] Shankarappa, R.K., Panneerselvam, A., Dwarakaprasad, R., Karur, S., Krishnanaik, G.B. and Nanjappa, M.C. (2009) Spontaneous asymptomatic coronary artery dissection in a young man. Journal of Cardiology, 54, 499502. doi:10.1016/j.jjcc.2009.03.007

[4] Pretty, H.C. (1931) Dissecting aneurysm of coronary artery in a woman aged 42. British Medical Journal, 1, 667.

[5] Jorgensen, M.B., Aharonian, V., Mansukhani, P. and Mahrer, P.R. (1994) Spontaneous coronary dissection: A cluster of cases with this rare finding. American Heart Journal, 127, 1382-1387.

doi:10.1016/0002-8703(94)90060-4

[6] Robinowitz, M., Virmani, R. and McAllister, H.A. (1982) Spontaneous coronary artery dissection and eosinophilic inflammation: A cause and effect relationship? American Journal of Medicine, 72, 923-928. doi:10.1016/0002-9343(82)90853-1

[7] Kearney, P., Erbel, R., Ge, J., Zamorano, J., Koch, L., Görge, G. and Meyer, J. (1994) Assessment of spontaneous coronary artery dissection by intravascular ultrasound in a patient with unstable angina. Catheterization and Cardiovascular Diagnosis, 32, 58-61. doi:10.1002/ccd.1810320114

[8] Ades, L.C., Waltham, R.D., Chiodo, A.A., et al. (1995) Myocardial infarction resulting from coronary artery dissection in an adolescent with Ehlers-Danlos syndrome type IV due to a type III collagen mutation. British Heart Journal, 74, 112-116. doi:10.1136/hrt.74.2.112

[9] Aldoboni Hamza, E.A., Majdi, K., Ngibzadhe, M., Palasaidi, S. and Moayed, D.A. (2002) Spontaneous dissection of coronary artery treated by primary stenting as the first presentation of systemic lupus erythematosus. Journal of Invasive Cardiology, 14, 694-696.

[10] Harada, H., Honma, Y., Hachiro, Y., Mawatari, T. and Abe, T. (2002) Traumatic coronary artery dissection. The Annals of Thoracic Surgery, 74, 236-237. doi:10.1016/S0003-4975(01)03515-9

[11] Steinhauer, J.R. and Caulfield, J.B. (2001) Spontaneous coronary artery dissection associated with cocaine use: A case report and brief review. Cardiovascular Pathology, 10, 141-145. doi:10.1016/S1054-8807(01)00074-6

[12] Azam, M.H., Roberts, D.H. and Logan, W.F.W.E. (1995) Spontaneous coronary artery dissection associated with oral contraceptive use. International Journal of Cardiology, 48, 195-198,. doi:10.1016/0167-5273(94)02238-E

[13] Ellis, C.J., Haywood, G.A. and Monro, J.L. (1994) Spontaneous coronary artery dissection in a young woman resulting from an intense gymnasium "work-out”. International Journal of Cardiology, 47, 193-194. doi:10.1016/0167-5273(94)90191-0

[14] Buys, E.M., Suttorp, M.J., Morshuis, W.J. and Plokker, H.W. (1994) Extension of a spontaneous coronary artery dissection due to thrombolytic therapy. Catheterization and Cardiovascular Diagnosis, 33, 157-160. doi:10.1002/ccd.1810330216

[15] Cheung, S., Mithani, V. and Watson, R. (2000) Healing of spontaneous coronary dissection in the context of glycoprotein IIb/IIIa inhibitor therapy: A case report. Catheterization and Cardiovascular Interventions, 51, 95-100. doi:10.1002/1522-726X(200009)51:1<95::AID-CCD22> 3.0.CO;2-M 